\title{
Suggested revision of the National High Blood Pressure Education Program blood pressure standardization for use in severely growth retarded children
}

\author{
Karlijn J. van Stralen • Kitty J. Jager • Enrico Verrina • \\ Franz S. Schaefer • Francesco Emma
}

Received: 17 November 2010 / Accepted: 25 November 2010/Published online: 31 December 2010

(C) The Author(s) 2010. This article is published with open access at Springerlink.com

Dear Sirs,

In 2007, the updated 4th report of the National High Blood Pressure Education Program was published [1] (http://www. nhlbi.nih.gov/health/prof/heart/hbp/hbp_ped.pdf). This report represents an international standard for the evaluation, diagnosis, and treatment of high blood pressure (BP) in the pediatric population and is based on data collected from 63,227 non-obese US children. BP measurements can be expressed as standard deviation scores (SDS) by subtracting the child's expected blood pressure, based on age and height percentiles, from the measured blood pressure and by dividing the resulting difference by one standard deviation. To correct BP for the height $z$ score, the authors have used fourth-degree polynomial regression models [2], also reported in appendix B of the report [1]. These models, one for each gender and for both systolic and diastolic blood pressures, are very useful to generate standardized

\footnotetext{
K. J. van Stralen $(\varangle) \cdot$ K. J. Jager

ESPN/ERA-EDTA Registry, Department of Medical Informatics, Academic Medical Center, University of Amsterdam, PO Box 22700,

1100 DE Amsterdam, The Netherlands

e-mail: K.J.vanStralen@amc.uva.nl

E. Verrina

Department of Paediatric Nephrology, Gaslini Children's

Hospital,

Genoa, Italy

F. S. Schaefer

Department of Paediatric Nephrology, University Hospital, Heidelberg, Germany

F. Emma

Division of Pediatric Nephrology, Bambino Gesú Hospital, Rome, Italy
}

measurements. They are often used both in clinical practice and in the context of clinical trials and constitute the basis on which blood pressure tables have been generated $[1,2]$. However, these models were developed on data from healthy children with expected normal heights, while children with grossly abnormal height $z$ scores $(<-6$ and $>6)$ were excluded.

In this letter we would like to emphasize the need to exercise caution when using these formulas in severely growth retarded children (i.e., height $z$ score below -3 ), such as children with end-stage renal disease, of in whom approximately $20 \%$ have height $z$ scores below -3 [3] and who are at particularly high risk of hypertension.

As shown in Fig. 1, the relation between height $z$ score and the expected BP calculated according to the published formula corrects appropriately BP when children have a normal or near-normal height. However, the fitting function displays an unexpected behavior for lower height $z$ score values. Obviously this is related to the use of complex fitting functions on a normal population. Computation of the predicted absolute BP in severely growth retarded children (e.g., boys with a height $z$ score $<-3.8$ for systolic BP, for example) yields unexpectedly high values, as illustrated in Fig. 1a. Figure 1b shows similar distortions for the computed BP SDS in both systolic and diastolic BP and in both genders. Hence, the use of these formulas in very short patients leads to an overestimation of reference values and consequently to the under-diagnosis of hypertension. For example, a 6year-old boy with a height of $90 \mathrm{~cm}$ (height $z$ score -4.9) and a systolic BP of $106 \mathrm{mmHg}$ is considered to be normotensive (BP SDS 1.5). If the same child is more growth retarded ( $85 \mathrm{~cm}$, for example) blood pressure will be interpreted to be even closer to the predicted mean value (BP SDS 1.0). Conversely, if height is slightly 

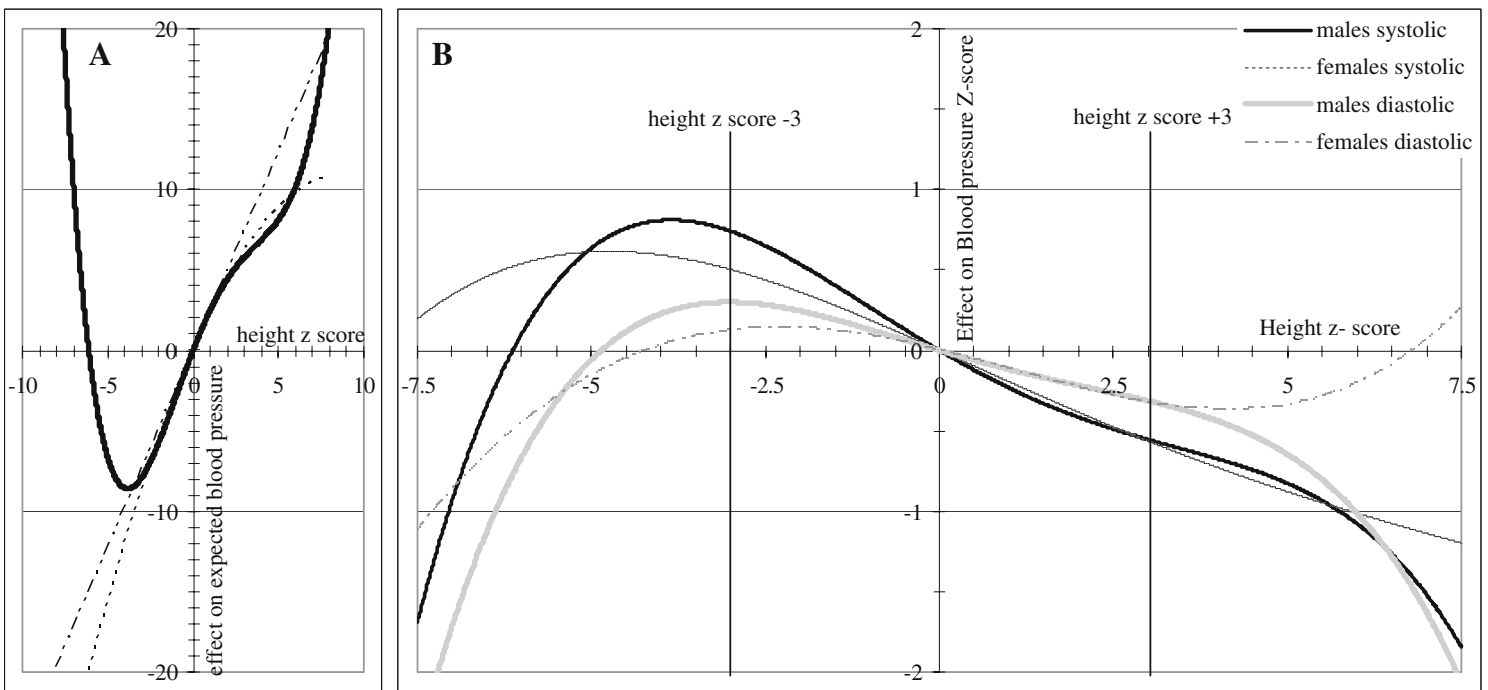

Fig. 1 a Relationship between height $z$ score and its effect on the expected systolic blood pressure for boys. b Relationship between height $z$ score and the blood pressure $z$ scores for both genders and systolic and diastolic blood pressure. All used the fourth-degree polynomial function as currently used for blood pressure standardization. When studying extremely low height $z$ scores, the polynomials suggest higher expected blood pressures instead of lower ones,

higher (96 cm, for example) he would be considered hypertensive.

To correct for this problem two simple solutions can be proposed:

(1) To correct BP data for height $z$ score only between -3 and +3 scores. Below and above these values, no further correction should be applied. This solution may be more prudent as no reference data are available for very short and very tall children.

(2) To use a simpler regression model. As stated by the authors of the fourth report, [2] "blood pressure levels appear to have an approximately linear function of height $z$ score for a given age-group", which is also illustrated in Fig. 1. The relation between BP and height $z$ scores could be recalculated using a linear or a quadratic line as shown in Fig. 1a.

In conclusion, the recently published formula for BP values correction for patients' height $z$ score is inappropriate for very short children. A revision is needed for their use in this resulting in lower blood pressure $z$ scores and therefore underdiagnosing the presence of hypertension. For comparison, dashed lines representing a linear $\left(2.46^{*}\right.$ height $z$ score $)$ and a quadratic $\left(-1.358 *\right.$ height $z$ score $^{2}+2.46 *$ height $z$ score $)$ function are also shown in $\mathbf{a}$, as well as the effects of having cut-offs at -3 and +3 height $z$ scores in $\mathbf{b}$

particular population of children, both in the clinical setting and for research purposes.

Open Access This article is distributed under the terms of the Creative Commons Attribution Noncommercial License which permits any noncommercial use, distribution, and reproduction in any medium, provided the original author(s) and source are credited.

\section{References}

1. National High Blood Pressure Education Program Working Group on High Blood Pressure in Children and Adolescents (2004) The fourth report on the diagnosis, evaluation, and treatment of high blood pressure in children and adolescents. Pediatrics 114:555-576

2. Rosner B, Cook N, Portman R, Daniels S, Falkner B (2008) Determination of blood pressure percentiles in normal-weight children: some methodological issues. Am J Epidemiol 167:653-666

3. U.S. Renal Data System (2008) Atlas of chronic kidney disease and end-stage renal disease in the United States. National Institutes of Health, National Institute of Diabetes and Digestive and Kidney Diseases, Bethesda, MD 